\title{
ON FLAT EXTENSIONS OF NOETHERIAN RINGS
}

\section{BIRGER IVERSEN}

In this note a ring will always mean a commutative, Noetherian ring with a unit.

Let $(B, \rho)$ be a flat extension of the ring $A$ (i.e. $B$ a ring and $\rho$ a ring-homomorphism of $A$ in $B$ such that $B$ regarded as $A$-module is flat), $M$ a finitely generated $A$-module and $N$ a submodule of $M$. The origin of this note is the study of primary decompositions of the $B$ submodule of $M_{B}=B \otimes_{A} M$ generated by $N$, when a primary decomposition of $N$ in $M$ is known. Without loss of generality I may specialize to $N=0$. The problem may now be restated in terms of the theory of injective modules, and the hypothesis that $M$ is finitely generated may fall: I shall study $I_{B}\left(M_{B}\right)$ (the injective hull of the $B$-module $M_{B}$ ), when $I_{A}(M)$ is known.

The key-result is that tensor product with respect to a flat $A$ module (regarded as functor in one variable) conserves essential monomorphisms. This fact reduces the problem to the case where $M=A / p$ ( $p$ a prime ideal in $A)$, and supplies the technique for the further treatment. The final result reduces the study of $I_{B}(B / B p)$ to that of simple numerical relations between three integers associated to a prime ideal in a ring. I conclude with an application to Macaulay and Gorenstein rings.

My references for the theory of injective modules are [5] or [4] and in what concerns localisation in this theory, [1]. Further I make use of the basic facts from [2].

Concerning flat modules I hardly make use of more than the definition. I could have used some results from "the ideal theory of flat extensions" as it is exposed in [3, Chapitre IV], or in [6], but since this "theory" is easily derived from the results of this note and since the needed results are easily proved by the technique developed here, I have stated and proved these results.

Notation. Let $A$ be a ring and $M$ an $A$-module and $p$ a prime ideal in $A . I_{\Delta}(M)$ denotes the injective hull of $M$. The cardinal number of the set of components isomorphic to $I_{A}(A / p)$ in a decomposition of $I_{A}(M)$ into a direct sum of indecomposable submodules is denoted $e_{p}(M)$. If $e_{p}(M) \neq 0$, I say that $p$ is associated to $M$, and if $p$ is minimal in the set of prime ideals associated to $M$, I say that $p$ is minimally associated to $M$. When no mistake is possible, I say that $p$ is associated to an ideal $a$ in $A$, when it is associated to $A / a$.

Received by the editors June 17, 1964. 
In the following $A$ denotes a (commutative, Noetherian) ring.

Theorem 1. Let $F$ be a flat $A$-module. If $f: M-N$ is an essential monomorphism, then $f \otimes l_{F}: M \otimes_{A} F \rightarrow N \otimes_{A} F$ is an essential monomorphism.

Proof. The proof consists in a successive reduction to the case where $A$ is a local, Artinian ring. In this case the theorem is obvious from the facts that a flat module is free [3, Chapitre II, $\$ 3, n^{\circ} 2$, Corollaire 2 de Proposition 5] and that a direct sum of essential monomorphisms is an essential monomorphism. All the homomorphisms considered in the proof are monomorphisms; hence we shall constantly identify with submodules using monomorphisms.

$1^{\circ}$ Reduction to the case where $M$ and $N$ are finitely generated $p$-primary modules (for a prime ideal $p$ in $A$ ).

The extension $N$ of $M$ is isomorphic to a subextension of the injective hull of $M$. Owing to the transitivity of essential extensions we may suppose that $N$ is the injective hull of $M$. Let $\left(N_{i}\right)_{i \in I}$ be a family of submodules of $N$ forming a direct sum decomposition of $N$ into indecomposable modules and let $M_{i}$ be a nonzero, finitely generated submodule of $M \cap N_{i}$. It is clear that the sum $\sum_{i \in I} M_{i}$ is direct and that $M_{i} \rightarrow N_{i}$ is essential since $N_{i}$ is injective and indecomposable. A new application of the transitivity of essential monomorphisms shows that it suffices to prove that the monomorphism $\left(\sum_{i \in I} M_{i}\right) \otimes F \rightarrow\left(\sum_{i \in I} N_{i}\right) \otimes F$ is essential. Since a direct sum of essential monomorphisms is an essential monomorphism, it now suffices to prove that $M_{i} \otimes F \rightarrow N_{i} \otimes F$ is essential, for each $i \in I$. But $N_{i} \otimes F$ is a union of submodules of the form $N_{i}^{\prime} \otimes F$, where $N_{i}^{\prime}$ is a finitely generated submodule of $N_{i}$ containing $M_{i}$. This remark completes the reduction.

$2^{\circ}$ Reduction to the case where $A$ is local and $M$ and $N$ are modules of finite length.

Suppose $M$ and $N$ are finitely generated $p$-primary modules, where $p$ is a prime ideal in $A$. Consider the commutative diagram

$$
\begin{gathered}
M_{p} \otimes_{\Delta} F \rightarrow N_{p} \otimes_{\Delta} F \\
\uparrow \\
M \otimes_{A} F \rightarrow N \otimes_{A} F .
\end{gathered}
$$

From the facts that $M$ and $N$ are $p$-primary and $F$ is flat, it follows that all the homomorphisms in the diagram are monomorphisms. It is trivially verified that the columns in the diagram are essential. From the transitivity of essential monomorphisms follows that it now 
suffices to prove that $M_{p} \otimes_{\Lambda} F \rightarrow N_{p} \otimes_{\Lambda} F$ is $A$-essential. This extension may be considered as an extension of $A_{p}$-modules and is as such isomorphic to the extension $M_{p} \otimes_{\Lambda_{p}} F_{p} \rightarrow N_{p} \otimes_{\Lambda_{p}} F_{p}$. To prove that this extension is $A$-essential, is to prove that it is $A_{p}$-essential. We may now finish the reduction by remarking that $F_{p}$ is a flat $A_{p}$-module.

$3^{\circ}$ Reduction to the case where $A$ is a local, Artinian ring.

Suppose that $A$. is local (its maximal ideal is designed by $m$ ) and that $M$ and $N$ are of finite length. Let $q$ be an $m$-primary ideal which annulates $M$ and $N$ (so one exists). The extension $M \otimes_{\Lambda} F \rightarrow N \otimes_{\Lambda} F$ may be regarded as an extension of $A / q$-modules and is as such isomorphic to the extension $M \otimes_{A / q}\left(A / q \otimes_{A} F\right) \rightarrow N \otimes_{\Delta / q}\left(A / q \otimes_{A} F\right)$. To prove that this extension is $A$-essential, is to prove that it is $A / q$ essential. Since $A / q \otimes_{A} F$ is a flat $A / q$-module, the reduction and hence the proof is completed.

Lemma 1. Let $p$ be a prime ideal in $A$ and $F$ a flat $A$-module. If $p F \neq F$, then $F / p F$ is a p-primary module.

Proof. $F / p F$ is obviously annulated by $p$. On the other side, multiplication in $F / p F \simeq F \otimes_{\Lambda} A / p$ with an element $s$ of $A$, not contained in $p$, is a monomorphism since multiplication with $s$ in $A / p$ is a monomorphism and $F$ is flat.

Proposition 1. Let $p$ be a prime ideal in $A, F$ a flat $A$-module and $M$ an $A$-module. Then

$$
e_{p}(M \otimes F)=e_{p}(M) \cdot e_{p}(F / p F) .
$$

Proof. According to Theorem 1, $I_{\Lambda}(M \otimes F)$ is isomorphic to $I_{\Lambda}(F \otimes I(M))$. Let $\left(E_{i}\right)_{i \in I}$ be a decomposition of $I_{\Lambda}(M)$ into a direct sum of indecomposable submodules. Since the injective hull of a direct sum of $A$-modules is isomorphic to the direct sum of the injective hulls of the modules in question, $I_{A}(M \otimes F)$ is isomorphic to $\bigoplus_{i \in I} I\left(E_{i} \otimes_{\Lambda} F\right)$. Let $p_{i}$ denote the prime ideal associated to $E_{i}$. Another application of Theorem 1 shows that $I_{A}\left(E_{i} \otimes F\right)$ is isomorphic to $I_{\Lambda}\left(\left(A / p_{i}\right) \otimes F\right) \simeq I_{\Lambda}\left(F / p_{i} F\right)$. Proposition 1 follows now from Lemma 1.

Corollary. Let $p$ be a prime ideal in $A, F$ a flat $A$-module and $M$ an $A$-module. Then $p$ is associated to $M \otimes_{A} F$ if and only if $p$ is associated to $M$ and $F \neq p F$.

In the rest of this paper $B$ denotes a ring and $\rho: A \rightarrow B$ a ring homomorphism with the property that $B$ regarded as $A$-module is flat.

When a $B$-module $N$ is regarded as $A$-module, it always turns upon 
the structure of $A$-module defined by $\rho$. For an $A$-module $M$, the $B$-module $B \otimes_{\triangle} M$ is denoted $M_{B}$.

If $p$ is an ideal in $A$, then the ideal in $B$ generated by $\rho(p)$ is denoted $B \rho(p)$. If $q$ is an ideal in $B$, the ideal $\rho^{-1}(q)$ is denoted $\hat{q} . B \rho(\hat{q})$ is denoted $\bar{q}$.

Lemma 2. Let $p$ be a prime ideal in $A$. If a prime ideal $q$ in $B$ is associated to $B / B \rho(p)$, then $\hat{q}=p$.

Proof. Suppose $q$ is associated to $B / B \rho(p)$. Then there exists an element $x \in B / p$ such that $q$ is the annulator of $x$. Considering $B / B \rho(p)$ as $A$-module, $x$ has the annulator $\hat{q}$. According to Lemma 1, $B / B \rho(p)$ considered as $A$-module is $p$-primary; hence $p$ is the only prime ideal in $A$ which is the annulator of some element of $B / B \rho(p)$. Therefore $p=\hat{q}$.

Remark. From Lemma 2 follows that if $p$ is a prime ideal in $A$ such that $B \rho(p) \neq B$ there exists a prime ideal $q$ in $B$ such that $\hat{q}=p$.

Proposition 2. Let $M$ be an $A$-module and $p$ a prime ideal in $B$. Then

$$
e_{p}\left(M_{B}\right)=e_{p}(B / \bar{p}) \cdot e_{\hat{p}}(M)
$$

Proof. As a simple corollary to Theorem 1 we have that the functor $M \Rightarrow M_{B}$ from the category of $A$-modules to that of $B$-modules conserves essential monomorphisms. Using this fact and Lemma 2 we can calculate a proof over that of Proposition 1. Indeed Proposition 1 and Proposition 2 could have been unified to a single proposition.

Proposition 3. Let $M$ be an $A$-module and $p$ a prime ideal in $B$. Then

(a) $p$ is associated to $M_{B}$ if and only if $\hat{p}$ is associated to $M$ and $p$ is associated to $\bar{p}$.

(b) $p$ is minimally associated to $M_{B}$ if and only if $\phi$ is minimally associated to $M$ and $p$ is minimally associated to $\bar{p}$.

Proof. (a) is an immediate consequence of Proposition 2.

(b) Suppose that $p$ is minimally associated to $M_{B}$. Since every prime ideal in $B$ which is associated to $\bar{p}$ is associated to $M_{B}$ according to (a), $p$ is minimally associated to $p . p$ is minimally associated to $M$ : Let $q$ be a prime ideal in $A$, contained in $p$ and associated to $M$. Since $B \rho(q)$ is contained in $p$, there exists a prime ideal $r$ in $B$, associated to $B \rho(q)$ and contained in $p$. (a) implies that $r$ is associated to $M_{B}$, hence $r=p$, and therefore (Lemma 2) $p=q$. Conversely, assume that $p$ is minimally associated to $\bar{p}$ and that $p$ is minimally associated to $M$. Let $q$ be a prime ideal in $B$, contained in $p$ and associated to $M_{B} . \hat{q}$ is then associated to $M$ and contained in $\hat{p}$, 
whence $\hat{q}=\hat{p}$. Further, $q$ is associated to $\bar{q}=\bar{p}$ and contained in $p$, hence $q=p$. Thus $p$ is minimally associated to $M_{B}$.

LEMMA 3. Let $p_{1}, \cdots, p_{n}$ be a strictly increasing sequence of prime ideals in $A$ and $q_{n}$ a prime ideal in $B$ such that $\hat{q}_{n}=p_{n}$. Then there exists an increasing sequence $q_{1}, \cdots, q_{n}$ of prime ideals in $B$ such that $\hat{q}_{i}=p_{i}$, for $i=1, \cdots, n$.

Proof. It obviously suffices to prove the lemma in the case $\boldsymbol{n}=\mathbf{2}$. $B \rho\left(p_{1}\right)$ is contained in $q_{2}$. A prime ideal $q_{1}$ contained in $q_{2}$ and associated to $B \rho\left(p_{1}\right)$ solves the problem according to Lemma 2.

Corollary. Let $a$ be an ideal in $A$ such that $B \rho(a) \neq B$. Then $\operatorname{alt}_{A}(a) \leqq \operatorname{alt}_{B} B \rho(a)$ (alt: altitude, height).

Remark. Let $M$ be an $A$-module and $p$ a prime ideal in $B$. Propositions 2 and 3 reduce the study of $e_{p}\left(M_{B}\right)$ and the relations " $p$ is associated to $M_{B}$ " and " $p$ is minimally associated to $M_{B}$ " to the case where $M=A / \hat{p}$.

Theorem 2 below solves these three problems in terms of three integers associated to a prime ideal in a ring. Let $C$ be a ring and $p$ a prime ideal in $C$ :

$1^{\circ} \operatorname{alt}_{c}(p)$ : is the altitude of $p\left(=\operatorname{Krull}-\operatorname{dim}\left(C_{p}\right)\right)$.

$2^{\circ} \operatorname{ass}_{C}(p)$ : the association-index of $p$. Let $\left(I^{n}(C)\right)_{n \in N}$ be a minimal injective resolution of the $C$-module $C$. $\operatorname{ass}_{C}(p)$ is the least integer $m$, for which $p$ is associated to $I^{m}(C)$. $\operatorname{ass}_{C}(p)$ is also the length of an arbitrary maximal prime-sequence $\left(C_{p}\right.$-sequence) in $C_{p}\left(=\operatorname{codim}\left(C_{p}\right)\right)$.

$3^{\circ} \operatorname{irr}_{c}(p)$ : the irreducibility-index of $p$. Set $\operatorname{ass}_{c}(p)=n$, then $\operatorname{irr}_{C}(p)=e_{p}\left(I^{n}(C)\right)$. If $a$ is an ideal in $C_{p}$ generated by a maximal prime-sequence, then $\operatorname{irr}_{C}(p)=e_{p_{p}}\left(C_{p} / a\right)$.

TheOREM 2. Let $p$ be a prime ideal in $B$. Then

$1^{\circ} \operatorname{alt}_{\Lambda}(\beta) \leqq \operatorname{alt}_{B}(p)$. $p$ is minimally associated to $\bar{p}$ if and only if $\operatorname{alt}_{\Lambda}(p)=\operatorname{alt}_{B}(p)$.

$2^{\circ} \operatorname{ass}_{\Lambda}(p) \leqq \operatorname{ass}_{B}(\hat{p}) ; p$ is associated to $p$ if and only if $\operatorname{ass}_{\Lambda}(p)=$ $\operatorname{ass}_{B}(p)$.

$3^{\circ}$ If $p$ is associated to $p$, then

$$
e_{p}(B / \bar{p}) \cdot \operatorname{irr}_{A}(\hat{p})=\operatorname{irr}_{B}(p) .
$$

Proof. From the homomorphism $\rho: A \rightarrow B$ we derive a local homomorphism $\rho^{\prime}: A_{\hat{p}} \rightarrow B_{p}$. $B_{p}$, considered as $A_{\hat{p}}$-module, is flat. Simple considerations now show that we may suppose that $A$ and $B$ are local rings with maximal ideal $\hat{p}$ respective $p$ and that $\rho$ is a local homomorphism.

The first part of $1^{\circ}$ is a special case of the corollary above. Suppose that $p$ is minimally associated to $\bar{p}$. Set alt $t_{\Delta}(\hat{p})=n$ and let $x_{1}, \cdots, x_{n}$ 
be a sequence of elements in $A$ which generate a $\hat{p}$-primary ideal. According to Proposition $3 \mathrm{~b}, p$ is minimally associated to the ideal generated by $\rho\left(x_{1}\right), \cdots, \rho\left(x_{n}\right)$. From the Altitude Theorem of Krull we get $\operatorname{alt}_{B}(p)=n$, hence $\operatorname{alt}_{B}(p)=\operatorname{alt}_{A}(\hat{p})$. The converse part follows immediately from the corollary to Lemma 3.

Let now $x_{1}, \cdots, x_{n}$ be a maximal prime sequence in $A, n=\operatorname{ass}_{\Lambda}(\hat{p})$. Since $B$ is a flat $A$-module, $\rho\left(x_{1}\right), \cdots, \rho\left(x_{n}\right)$ is a prime-sequence in $B .2^{\circ}$ follows now immediately from Proposition $3 a$, and $3^{\circ}$ follows from Proposition 2 posing $M=$ the residue ring of $A$ with respect to the ideal generated by $x_{1}, \cdots, x_{n}$.

REMARK. Suppose that $A$ and $B$ are local rings with maximal ideal $m$ respective $n$. Then the relation " $B \rho(m)$ is $n$-primary" is either true for all flat, local homomorphisms of $A$ in $B$ or false for all such homomorphisms. $e_{n}(B / B \rho(m))$ is independent too of the flat, local homomorphism considered.

Let $C$ be a ring. $C$ is a Macaulay ring if and only if $\operatorname{ass}_{C}(p)=\operatorname{alt}_{C}(p)$ for all prime (resp. maximal) ideals $p$ in $C$. $C$ is a Gorenstein ring if and only if $C$ is a Macaulay ring for which $\operatorname{irr}_{C}(p)=1$ for all prime (resp. maximal) ideals in $C$.

From these remarks, for which I shall refer to [2], we get the following corollaries to Theorem 2 .

Corollary 1. Suppose $B \rho(m) \neq B$ for each maximal ideal $m$ in $A$, and let $p$ be a prime ideal in $A$. Then:

$1^{\circ}$ If $B$ is a Macaulay ring then so is $A$ and any prime ideal in $B$ associated to $B \rho(p)$ is minimally associated.

$2^{\circ}$ If $B$ is a Gorenstein ring then so is $A$ and all the components in a primary decomposition of $B \rho(p)$ in $B$ are irreducible.

Corollary 2. Let $A$ and $B$ be local rings with maximal ideal $m$ respective $n$ and $\rho: A \rightarrow B$ a flat, local homomorphism, and suppose that $n$ is generated by $\rho(m)$. Then $A$ is a Gorenstein ring if and only if $B$ is a Gorenstein ring.

\section{REFERENCES}

1. H. Bass, Injective dimension in Noetherian rings, Trans. Amer. Math. Soc. 102 (1962), 18-29.

2. - On the ubiquity of Gorenstein rings, Math. Ann. 82 (1963), 8-28.

3. N. Bourbaki, Algebre commutative, Hermann, Paris, 1961; Chapitres I-IV.

4. P. Gabriel, Des categories abeliennes, Bull. Soc. Math. France 90 (1962), 323448.

5. E. Matlis, Injective modules over Noetherian rings, Pacific J. Math. 8 (1958), 511-528.

6. M. Nagata, Local rings, Wiley, New York, 1962.

University of Copenhagen, Demmark 